\title{
LOS DEPARTAMENTOS
}

\section{FISICA}

El Departamento de Física ofrece los programas de LICENCIATURA EN FISICA y MAESTRIA EN DOCENCIA DE LA FISICA.

Durante este período ha presentado un Proyecto dirigido a docentes en ejercicio, "Especialización de Docencia de las Ciencias para el Nivel Básico", el cual ha obtenido su aprobación en las primeras instancias de la Universidad y espera ofrecerlo para el Primer Semestre de 1992.

El grupo Física y Cultura viene desarrollando el programa "Los Lunes de la Enseñanza de la Física" con la participación de algunas personas y grupos de trabajo de otras instituciones con intereses afines a la divulgación de la Cultura Científica y enseñanza de las Ciencias. El grupo prepara el tercer número de la revista FISICA y CULTURA, cuya publicación se hará antes de finalizar el presente año.

El Departamento ha participado activamente en el VI Coloquio sobre la enseñanza de la Física y en el XIV Congreso Nacional de Física.

El grupo de estudiantes denominado El Tambor de Feynman publicó el segundo número de su revista. El grupo de estudiantes Aldebarán identificado con los propósitos de los astrónomos aficionados, se trasladó a la ciudad de Neiva para presenciar y participar de las actividades científicas en torno al Eclipse Solar.

\section{EDUCACION FISICA}

El Departamento de Educación Física de la Universidad Pedagógica Nacional, es el departamento más antiguo en el país como formador de Licenciados en Educación Física. Actualmente funcionan más departamentos en el país.

El Licenciado en Educación Física termina con una formación que le permite desempeñarse como profesor en primaria y secundaria, entrenador en equipos deportivos, administrador en instituciones que promueven el deporte, la Educación Física y la recreación; como recreacionistas, rehabilitador, preparador físico, instrucción de formación deportiva, promotor de actividades deportivas, arbitraje y líder en general de las actividades del campo deportivo y recreativo.

El Departamento de Educación Física adelanta sus programas en la sede de la calle 73 No.11-69, pero por convenio utiliza otros escenarios en COLDEPORTES, LA JUNTA ADMINISTRADORA DE DEPORTES DE SANTA FE DE BOGOTA, la Universidad Nacional y TELECOM. Actualmente el Departamento adelanta un currículo cuya última reforma se suscitó en 1984. El nuevo currículo maneja su eje central con base en el Estudio del Movimiento Humano, el Estudio del Hombre y la Relación Hombremovimiento.

El Departamento de Educación Física de la UPN es el pionero en el montaje del Deporte obligatorio para todos los estudiantes de un centro docente. Es así como en la UPN todo estudiante debe cursar obligatoriamente unas ULAS en Deporte en dos niveles, para 
optar su título universitario; el objetivo primordial es dar a conocer a profesionales de otras carreras los verdaderos valores del deporte como medio educativo y formativo de la juventud y a la vez formar los futuros profesionales con hábitos propios sobre la práctica diaria del deporte y sus beneficios.

El Departamento cuenta además con una piscina terminada en 1980, alrededor de la cual se desarrolla una nueva metodología de la enseñanza de la natación, y se da servicio abierto a la comunidad. Como este proyecto, el Departamento adelanta nueve programas de investigación en diferentes ternas: Salud y Educación Física, Deporte de Alto Rendimiento, Pedagogía y Didáctica, etc.

El Departamento adelanta el estudio de factibilidad sobre la apertura de tres Post-grados: a) Entrenamiento; b) Pedagogía y Didáctica y c) Administración Deportiva. Paralelamente, y en conjunto con COLDEPORTES, el COMITE OLIMPICO COLOMBIANO y el CENTRO DE INVESTIGACIONES EN DEPORTE, se estudia el montaje de la Red Nacional de Informática en Deporte, con la asesoría de la Universidad Deportiva de España y el Instituto Alemán de Investigaciones en Deporte.

De acuerdo con los estudios de reforma en que actualmente se encuentra la Universidad, se iniciaron los estudios para la apertura de un nuevo programa de Pregrado y una reforma curricular al programa actual para llegar a la configuración de un nuevo profesional que responda a la apertura económica y los cambios que ésta genera en los campos culturales y educativos.

\section{MATEMATICAS}

El Departamento de Matemáticas de la Universidad Pedagógica Nacional desarrolla en la actualidad dos programas de formación: la Licenciatura en Matemáticas y el Magister en Docencia de la Matemática.

Con el fin de responder en mejor forma a las demandas del sector educativo y de organizar un trabajo de extensión que aporte al mejoramiento y capacitación de los docentes en ejercicio, se está tramitando la aprobación de un énfasis en computación para la carrera de Licenciatura en Matemáticas y de una Especialización en Educación de la Matemática.

En el presente año, la actividad académica del Departamento se ha enriquecido con la realización de algunos eventos que se reseñan a continuación:

\section{ASESORIA INTERNACIONAL}

En el lapso comprendido entre el 5 y el 14 de agosto de 1991, dentro de la ejecución del convenio ICFES-BID para el mejoramiento de la capacidad de investigación, visitó el Departamento de Matemáticas de la Universidad Pedagógica Nacional, el Dr. Joseph M. Fortuny, coordinador del Doctorado en Didáctica de la Matemática y de las Ciencias Experimentales de la Universidad Autónoma de Barcelona.

El doctor Fortuny brindó su asesoría al equipo de profesores que prepara el programa de postgrado en Didáctica de la Matemática a nivel de especialización y al grupo de investigadores que trabaja en el proyecto "Construcción de un modelo pedagógico, curricular, metodológico y didáctico para la enseñanza de la matemática en el nivel 
secundario". Además, el profesor Fortuny contribuyó a la preparación del Encuentro de Geometría y sus Aplicaciones con el desarrollo de las siguientes actividades:

- Curso sobre "Estrategias para la educación geométrica en secundaria"

- Conferencias:

1- $\quad$ La Evaluación del aprendizaje en matemáticas

2- La geometría en la educación y la cultura

3- Ciencia cognitiva y educación matemática

A las actividades desarrolladas por el profesor Fortuny asistieron profesores de varias universidades y colegios de Santa Fe de Bogotá.

\section{ENCUENTRO DE GEOMETRIA Y SUS APLICACIONES}

Este evento fue coordinado por el Postgrado en Docencia de la Matemática de la Universidad Pedagógica Nacional y el Departamento de Matemática de la Universidad de los Andes, y se desarrolló entre el 23 y el 27 de septiembre de 1991.

Los objetivos del evento fueron esencialmente: la divulgación de los trabajos de investigación que se adelantan en las universidades del país, en relación con la Geometría y sus Aplicaciones, la formación de grupos de estudio y de investigación a nivel nacional alrededor de la geometría, la motivación a los profesores de secundaria y de universidad para que estudien el punto de vista geométrico en los temas tratados en su ejercicio docente.

En el desarrollo del evento se realizaron cursillos sobre divulgación y aplicaciones pedagógicas, conferencias sobre ternas de investigación y talleres sobre didáctica. Se contó con la participación de destacados docentes e investigadores de la UPN, Universidad de los Andes, Universidad Nacional, Universidad Distrital, Universidad del Valle, Universidad Industrial de Santander, Universidad Pedagógica y Tecnológica de Colombia y Universidad Javeriana. Se contó con la asistencia de 100 participantes, profesores universitarios, de colegios de secundaria y estudiantes de carreras de licenciatura.

\section{COLOQUIO DISTRITAL DE MATEMATICAS Y ESTADISTICA}

Entre el 2 y el 6 de diciembre de 1991, la Universidad Pedagógica Nacional será sede del $8^{\circ}$. Coloquio Distrital de Matemáticas y Estadística, evento que se realiza cada año con la coordinación de las Universidades Nacional, Distrital y Pedagógica.

Para este año se espera la participación .de más de 300 profesores de matemáticas de todos los niveles del sistema educativo, quienes tendrán la posibilidad y la oportunidad de realizar un intercambio académico con sus colegas y de actualizarse en lo relativo a los avances de la disciplina matemática y su pedagogía.

Se han programado 30 cursillos sobre diferentes tópicos y 15 conferencias, todas ellas bajo la orientación de destacados docentes e investigadores de las universidades de Santa Fe de Bogotá y de algunas otras capitales. 